\title{
Cheap optical transducers (CHOTs) for narrowband ultrasonic applications
}

\author{
T Stratoudaki, J A Hernandez, M Clark and M G Somekh \\ School of Electrical and Electronic Engineering, University of Nottingham, Nottingham, \\ NG7 2RD, UK \\ E-mail: T.Stratoudaki@nottingham.ac.uk
}

Received 5 October 2006, in final form 17 December 2006

Published 5 February 2007

Online at stacks.iop.org/MST/18/843

\begin{abstract}
We have developed an innovative ultrasonic transducer system (CHOT)

which is optically excited by means of lasers. It can be used both for generation and detection of narrowband ultrasound and provides non-contact (or even remote), couplant-free generation and/or detection. It has also the advantage of being inexpensive to manufacture and the simplicity of its concept makes it ideal for industrial applications. In this study we present results where CHOTs have been used both for excitation and detection of surface acoustic waves. An initial theoretical model is also presented which describes the principle of operation.
\end{abstract}

Keywords: transducers, ultrasound, narrowband, laser

\section{Introduction}

Using ultrasound for the testing of materials is a powerful, well-established and relatively simple technique: ultrasound propagates into the inner structure or onto the surface of the materials giving information about their properties, thickness and possible defects, to name but a few of the current applications.

Ultrasound is usually generated and detected by contact devices such as piezoelectric transducers. However, there are certain disadvantages associated with conventional transducers. First of all they generally require a coupling medium in order to transfer ultrasound to the inspected material. Coupling may be wet or dry but it introduces many problems to the inspection system. These can be grouped in problems associated with scanning large surfaces (e.g., drying of couplants or sensitivity variations from spot to spot), or limited temperature ranges which is particularly important for industrial applications. Apart from the necessity of a coupling medium for conventional transducers there are also other problems related to the weight of the transducer itself which might influence the measurements or the inspected structure, sometimes there are size considerations to be taken into account and also problems presented by unusual geometries and places with difficult access. Remote operation is preferable, particularly in the case of hostile environments, such as extreme temperatures.
For all the above reasons, we have concentrated our efforts in making a new ultrasonic transducer system which is optically activated both for generation and detection and that would address all the above-mentioned shortcomings of the currently available transducers. CHOTs (cheap optical transducers) are structures that can be printed or attached onto the surface of the sample and consist of two parts: the gCHOT (for generation) and the d-CHOT (for detection). They can be used as a coupled system or separately to either generate or detect frequency-controlled ultrasound that is respectively detected or generated by other means.

CHOTs use lasers to excite and detect thus bringing the advantages of laser-based ultrasound in the narrowband frequency range. For the results presented in this paper, a q-switched Nd:YAG pulsed laser emitting at $1064 \mathrm{~nm}$ wavelength was used for ultrasonic generation and a CW HeNe laser emitting at $633 \mathrm{~nm}$ wavelength was used for detection. The choice of laser for ultrasonic generation was made based on the fact that Nd:YAG lasers are well-adapted high power lasers for this application, are available in portable form and are becoming less expensive. They can be fibre coupled to answer laser safety problems. The HeNe laser used for detection is a commonly found medium power laser that can also be fibre coupled.

An example of a simple CHOT structure consisting of patterns deposited on the sample and used for generation and detection of surface acoustic waves (SAWs) is presented in this 


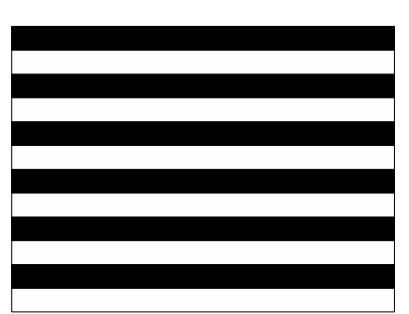

(a)

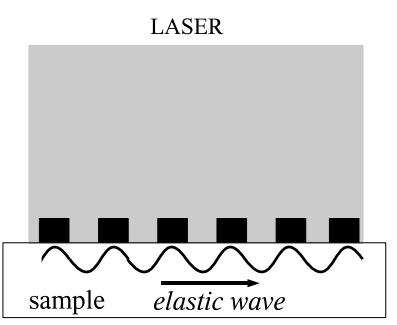

(b)
Figure 1. Simple g-CHOT structure consisting of a pattern deposited on the sample for generation of surface waves: $(a)$ the substrate (white lines) is transparent/absorbing to laser radiation, the deposited layer (black lines) is respectively absorbing/reflective, i.e. generation of laterally modulated thermal stress; $(b)$ cross section of the sample. In this case, the illuminated area (laser spot size) is larger than the g-CHOT.

paper. However, they can be used for generation/detection of other wavemodes as well as to control the directivity and frequency content of the waves simply by changing their geometrical features. We used BK7 glass samples for inspection to demonstrate the principle.

\section{Theory}

The following subsections describe the theory behind the operation of the g-CHOT, the d-CHOT but also of the combined CHOT system for ultrasonic generation and detection. Concerning the g-CHOT, examples of simple patterns have been 'drawn' on to the samples for excitation of narrowband SAWs in the $\mathrm{GHz}$ regime [1-4]. However, by changing the geometrical characteristics of the g-CHOT structure one can control the mode and frequency content of the generated wave as well as its directivity. There is also a clear need for an equivalent narrowband detection arrangement with the advantages offered by laser ultrasonics and that is the d-CHOT. The d-CHOT is a novel device consisting of a structure on the surface of the sample acting as a reflective grating and which, activated by a laser beam, is able to detect and filter ultrasound. The unique advantages of the d-CHOT are best exploited when both CHOTs are brought together to form a single unit, the CHOT system, for ultrasonic generation and detection. Such configuration offers high sensitivity, stability and ease of use combined with potential low manufacturing costs that make this application so important for the nondestructive testing community.

\section{1. $g$-CHOT}

Ultrasound is generated when the light emitted by a pulsed laser is absorbed by the material. In the low laser power thermoelastic regime there is no damage of the material and the process is nondestructive. The laser beam incident to the sample locally heats its surface and causes it to expand rapidly at times that are comparable to the rise time of the laser pulse which - for such applications - is typically in the order of $10-20$ ns.

The idea behind the making of the $\mathrm{g}$-CHOT is to create an ultrasonic source with an appropriately high contrast between absorbing and non-absorbing regions of the irradiated sample

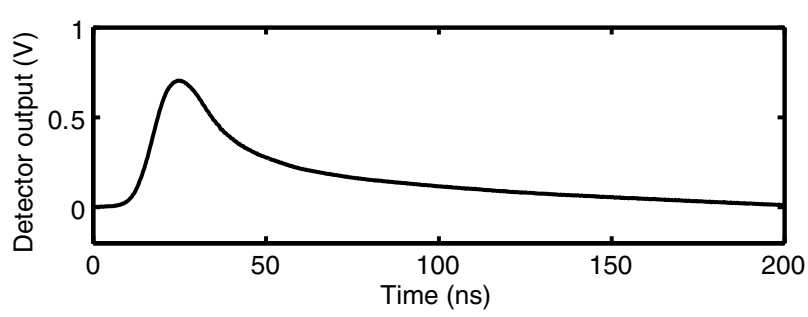

(a)

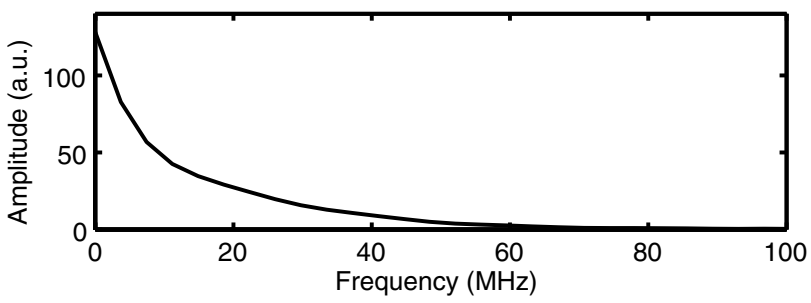

(b)

Figure 2. Nd:YAG laser: $(a)$ temporal profile. $\mathrm{FWHM}=20 \mathrm{~ns}$, rise time $=9 \mathrm{~ns},(b)$ Frequency spectrum of the pulse.

[1]. An example of such a structure is shown in figure 1 . This is the pattern that was used to generate plane SAWs of a chosen frequency in our experiments. The black lines represent areas where laser radiation is highly absorbed and the white lines represent areas where laser radiation is either transmitted or reflected. Therefore, g-CHOTs depend strongly on the material's properties. For example, the samples that we used for our experiments were made of BK7 glass. This material was chosen because it is highly transparent to $1064 \mathrm{~nm}$ radiation emitted by our Nd:YAG laser (according to the manufacturer, $94 \%$ of the incident energy is transmitted through the $8 \mathrm{~mm}$ thick sample while $4 \%$ is reflected). We then created a pattern of aluminium stripes with appropriate thickness corresponding to the ultrasonic frequency that we wanted to generate. Aluminium absorbs $1064 \mathrm{~nm}$ laser radiation more strongly than the transparent substrate and thus creates the necessary absorption contrast for the g-CHOT. In fact, aluminium reflects $93 \%$ of the incident energy [5] while the remaining $7 \%$ is absorbed within the electromagnetic skin depth which is of the order of tens of nanometres. Even though the absorption contrast is not high and most of the laser energy is reflected by the g-CHOT, it was still sufficient to generate detectable ultrasound.

Laser ultrasound is usually associated with broadband generation. The bandwidth of the generated signal is related to the bandwidth of the laser pulse. For our experiments, the laser pulse and its frequency content can be seen in figures 2( $a$ ) and $2(b)$. It can be seen that the pulse duration of the laser is $\sim 18 \mathrm{~ns}$ and its spectrum extends up to $\sim 30 \mathrm{MHz}$. However, for our applications we wanted to generate narrowband ultrasound. The bandwidth selection is done by appropriate tailoring of the thickness of the lines of the illuminated pattern [2].

More specifically, let us assume a single focused line (of infinitely small width) on the sample surface, of a laser beam with an infinitely short time duration (i.e., a delta pulse). It can be shown that, as long as the laser energy is absorbed in a layer much thinner than the ultrasonic wavelength (as is our 


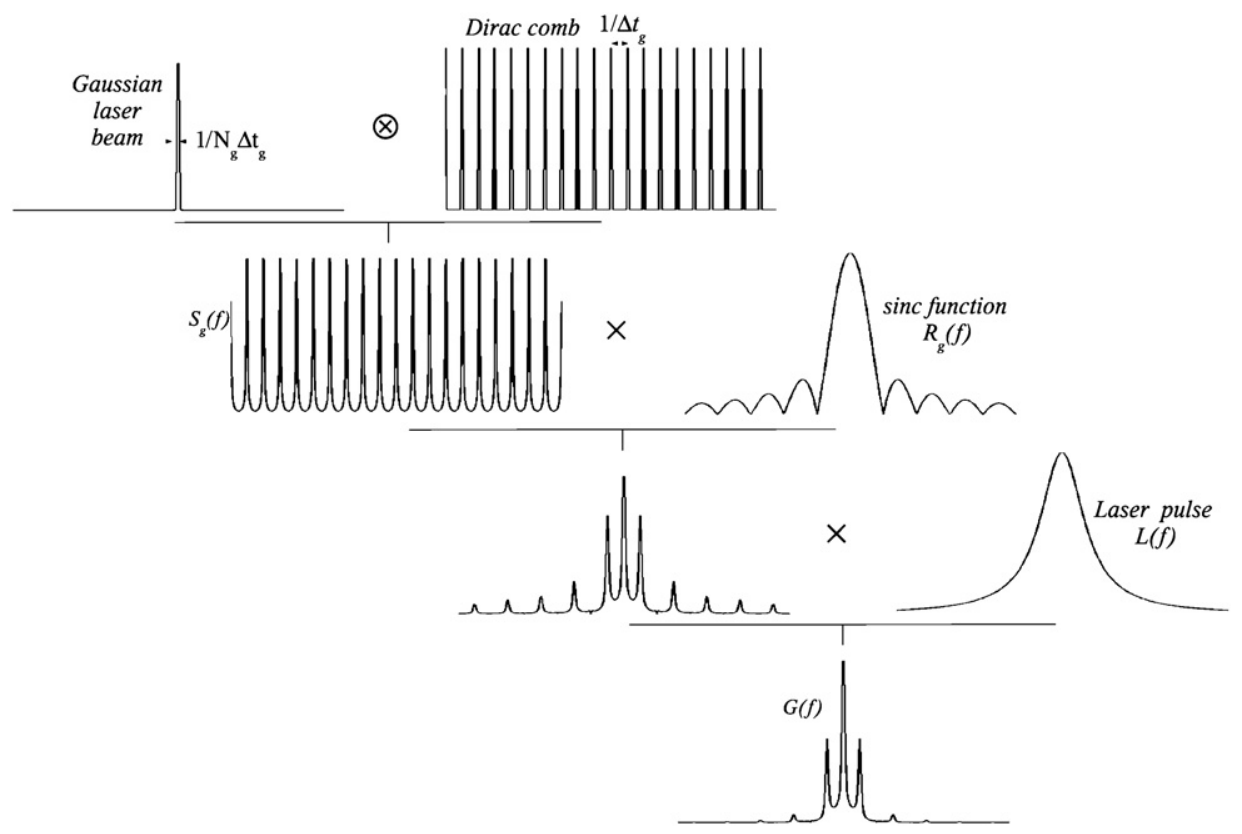

Figure 3. Graphic representation of the frequency filtering action of the g-CHOT.

case), the generated wave depends on the spatial and temporal characteristics of the laser pulse.

To model the generation, we first assume a Dirac comb, i.e. an infinite number of delta functions with spacing of width $\Delta t_{g}$, denoting the characteristic time that it takes for the acoustic wave to propagate between two adjacent lines and which is related to the spacing between the individual lines of width $w_{g}$ and the surface wave velocity $c$. It follows that the frequency content of the generated ultrasound would be another Dirac comb where the adjacent lines would have a spacing of $1 / \Delta t_{g}$. Since the number of lines in the g-CHOT pattern is not infinite but $N_{g}$, we can represent this by multiplying the Dirac comb with a top hat function that will include $N_{g}$ lines. This action would correspond to a convolution in the frequency domain. The laser beam intensity profile is usually approximated with a Gaussian distribution instead of a top hat and we will consider this to be the case here, while its width would be $N_{g} \Delta t_{g}$. In the frequency domain this Gaussian would correspond to another Gaussian with a width of $1 / N_{g} \Delta t_{g}$. The result from its convolution with the Dirac comb is the function $S_{g}(f)$ depicted in figure 3. Again, the individual lines of the gCHOT pattern are not infinitely small but have a width $w_{g}$ and we can further assume that each one has a top hat spatial profile (i.e., a sinc function $R_{g}(f)$ in the frequency domain). In order to include this we have to convolve the initial Dirac comb with the line profile. In the frequency domain this corresponds to a multiplication of $S_{g}(f)$ with $R_{g}(f)$. In this analysis we have assumed that the laser pulse is infinitely short in time. If, in addition we consider the temporal profile of the laser, then we would have to convolve the laser pulse with the spatial profile of the previous analysis. In the frequency domain this is equal to a multiplication between the previous result and the frequency content of the laser pulse $L(f)$. The result is shown as $G(f)$ in figure 3 (for a more rigorous mathematical analysis the reader is referred to $[6,7])$.

For a Gaussian beam with pulse duration of $20 \mathrm{~ns}$ (i.e., a rise time of $\sim 10 \mathrm{~ns}$ ), the results from a single line and an array

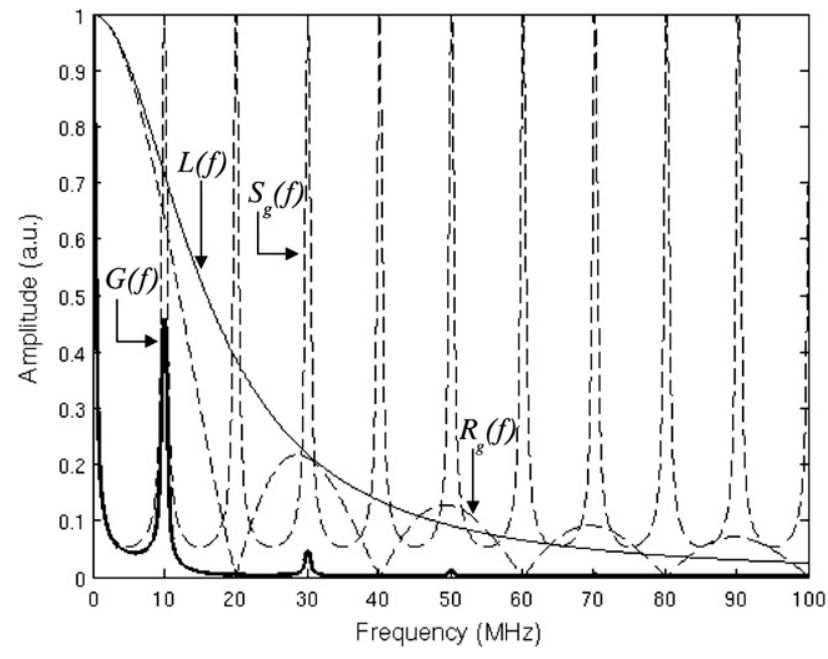

Figure 4. g-CHOT: theoretical spectra of ultrasound generated from an array of $N_{g}=10$ lines $(G(f))$ with appropriate width and spacing for generation of $10 \mathrm{MHz}$ ultrasound in BK7 glass and comparison with the frequency content of a laser pulse $(L(f))$ with rise time of 10 ns. The functions $S_{g}(f)$ and $R_{g}(f)$, as defined in figure 3, are also represented. All spectra have been normalized to unity.

of 10 similar lines with a thickness of $168 \mu \mathrm{m}$ and equally spaced can be seen in figure 4 . In this case we assumed a material with surface wave velocity $3370 \mathrm{~m} \mathrm{~s}^{-1}$ (corresponding to the velocity measured for BK7 glass) and the chosen array spacing results in generating ultrasonic signals of $10 \mathrm{MHz}$ frequency content. It can be seen that, whereas one line has a broadband response, an array of lines produces narrowband signals (see also [6, 8, 9]). The bandwidth of the generated ultrasound depends on the number $N_{g}$ of g-CHOT's lines that are illuminated by the laser pulse and the larger this number is, the narrower the bandwidth (figure 5).

The above theory for generation of narrowband ultrasound has been used in the past, however all the previous techniques 


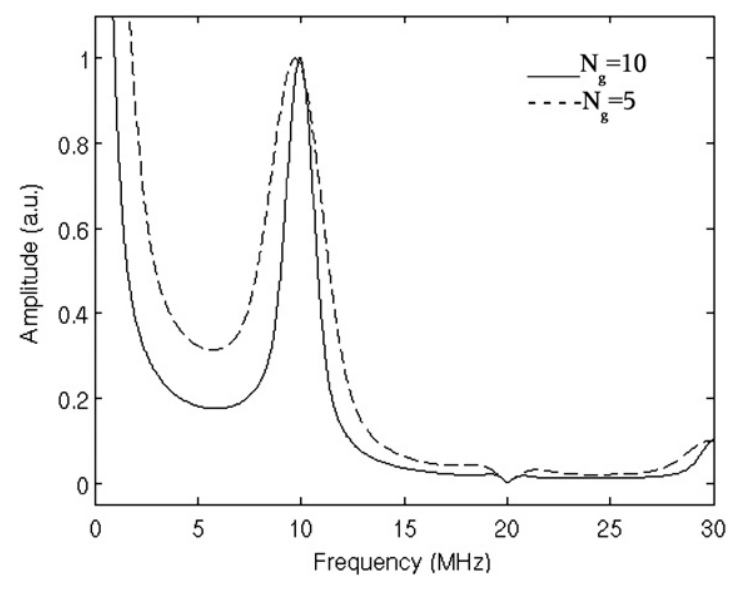

Figure 5. g-CHOT: theoretical spectra of ultrasound generated from an array of $N_{g}=10$ lines (solid) and $N_{g}=5$ lines (dashed) assuming a line width and spacing appropriate for generation of $10 \mathrm{MHz}$ ultrasound on BK7 glass.

required temporal and spatial tailoring of the laser beam. For the spatial modification of the beam in particular, various methods have been used: masks to partially block the beam [10], lenticular arrays [11, 12], an array of optical fibres [7, 8], diffraction gratings [6], the interference pattern of two laser beams [13-16], holograms [17] or a spatial light modulator [18]. However, most of these systems suffer from one or more of the following: they are expensive, require careful alignment and knowledge of optics, may be bulky, need a highly experienced operator and are difficult to adapt in an industrial environment. As for modifying the sample surface itself, as mentioned at the beginning of this section, some groups have used simple patterns to generate narrowband SAWs in the GHz regime [1-4]. However, the present paper extends this idea to offer increased control of the generated ultrasound characteristics especially when combined with an appropriate detection system (d-CHOT).

With g-CHOTs, instead of modifying the laser beam, the sample surface is slightly modified to provide the necessary light absorption contrast. The laser is a typical q-switched $\mathrm{Nd}$ :YAG, all the optics involved are very simple and the beam can be delivered via a fibre. In addition, the user has a choice of generating frequencies which is only limited by the bandwidth of the laser pulse. For example, the laser used in this study had a bandwidth between 0 and $30 \mathrm{MHz}$ (as can be seen in figure 2). By changing the spacing of the absorbing lines on the g-CHOTs pattern we were able to choose between 5,10 and $20 \mathrm{MHz}$ narrowband ultrasonic generation.

\section{2. $d-C H O T$}

Since the g-CHOT is based on laser-generated ultrasound, it is best to limit the bandwidth of the generation and also the detection system (provided that no useful signal is lost in the process) in order to increase the signal-to-noise ratio and the sensitivity of the system [12]. This is particularly useful for applications requiring narrowband ultrasonic signals such as the measurement of the low amplitude harmonics in nonlinear ultrasonics. During the development of a laser-based system it is often preferable for the detection to be based on lasers as

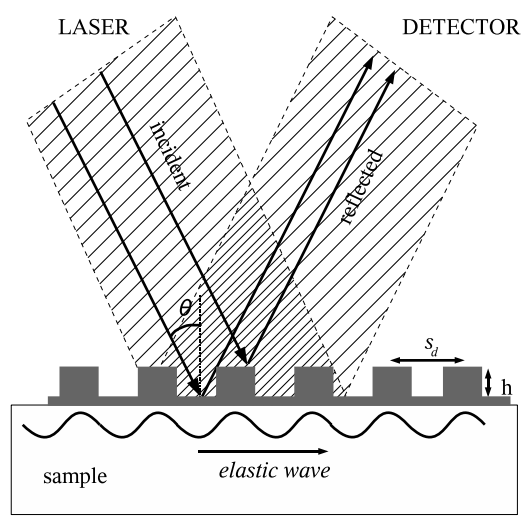

Figure 6. d-CHOT pattern (cross section): reflective material (in grey) with appropriate height $(h)$ and spacing $\left(s_{d}\right)$ to detect ultrasonic surface waves.

well, since this method is also non-contact and couplant-free. For these reasons we concentrated our efforts on developing a new detection system (d-CHOT) that would make best use of the g-CHOT. However, this highly sensitive optical transducer can be used on its own to detect and filter ultrasound generated by any other methods.

The d-CHOT consists of a structure on the surface of the sample, which reflects light. It is in effect a reflective grating. The grating consists of appropriately designed steps, which introduce the desired path difference to the light (figure 6). Again, the operation is remote and the actuator is a laser, which simply illuminates the area of the grating. The beam reflected by the d-CHOT is separated into various diffraction orders and the main reflection (zero order). The latter is separated from the rest by means of an iris and directed onto the photodetector. The height of the steps $(h)$ of the d-CHOT for normal incidence corresponds to $1 / 8$ th of the optical wavelength. In our case, we were using a medium power $\mathrm{CW}$ HeNe laser, emitting at $633 \mathrm{~nm}$ wavelength, i.e. the height of the steps should be $\sim 79 \mathrm{~nm}$. For this configuration, $50 \%$ of the incident laser energy is directed to the zero order. When the generated ultrasound travels below the area of the d-CHOT, this height changes, thus modulating the amount of energy directed to the zero order. It is this modulation that is detected by the photodiode. In effect, each step of the d-CHOT is a miniature common path interferometer. Its advantages however are that, because the effective path difference between the 'signal beam' and the 'reference beam' (referring to the reflection from the top and the bottom of the step) is of the order of $1 / 4$ of the wavelength of the detection laser, it is very stable and can be easily adapted into noisy industrial environments. In addition, because it makes use of the direct specular reflection (zero order) of the grating, it can be used with samples that are not rigorously polished as long as there is enough light to provide a detectable signal. At the same time, the d-CHOT filters the detected ultrasonic signal. The spacing of its lines corresponds to the distance travelled by the ultrasonic wave of the desired frequency. For example, in our case, we were generating surface waves on BK7 glass and we wanted to detect ultrasound at $f_{d}=5,10$ and $20 \mathrm{MHz}$. The spacing of the grating was $s_{d}=c / f_{d}$. In this way the d-CHOT filters the ultrasound with the appropriate frequency providing narrowband detection. 


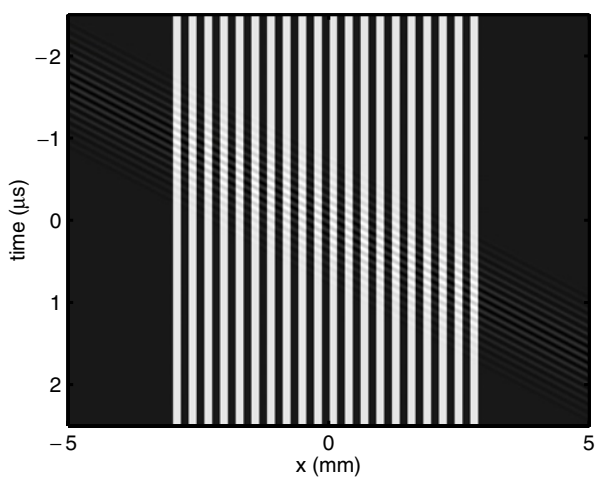

(a)

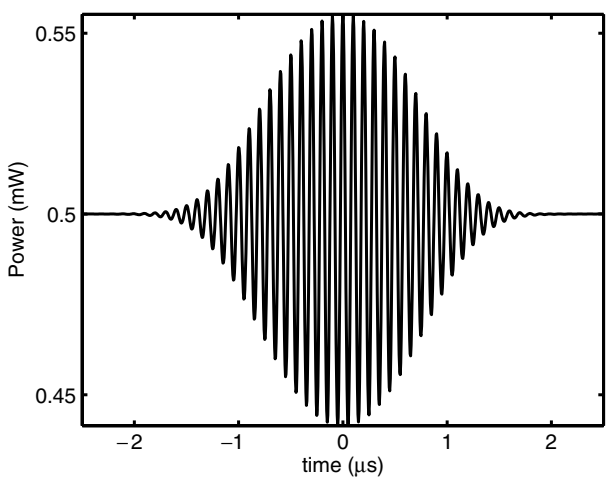

(b)

Figure 7. Optical detection of ultrasonic signal from the d-CHOT: $(a)$ phase of the optical field leaving the d-CHOT, $(b)$ optical power into the zero order for SAW of $10 \mathrm{~nm}$ amplitude.

Following a similar analysis with the g-CHOT, if one assumes that a broadband source (such as a laser focused onto a single line) generates ultrasound, then the frequency spectrum $D(f)$ of the signal detected by the d-CHOT would be the convolution between a Dirac comb and a Gaussian function representing the Gaussian function that includes $N_{d}$ lines and another multiplication with a sinc function corresponding to the top hat spatial profile of each individual line of the dCHOT grating. As with the g-CHOT, the bandwidth of the peaks of $D(f)$ depends on the number of illuminated d-CHOT lines and the larger the number of illuminated lines, the narrower the bandwidth. In this way, the d-CHOT has a great potential for high efficiency ultrasonic detection.

To model the signal detection of a plane SAW using the d-CHOT, we first assume it to have the spatial characteristics of a square grating with $N_{d}$ lines. We also assume that only the zero diffracted order reaches the detector. Both these assumptions are easy to realize in practice. The zero order is the dc component of the reflected field or averaged field. We will first assume normal incidence $\left(\theta=0^{\circ}\right)$ and that the reflectivity of the grating is uniform. The reflected field can be represented as

$$
s(t)=\overline{\mathrm{e}^{\mathrm{i} 0} \mathrm{e}^{2 \mathrm{i} k g(x)} \mathrm{e}^{2 \mathrm{i} k u(x-c t)}} .
$$

The first term on the right side of equation (1) represents the zero diffracted order, the second is the contribution of the grating and the third is due to the ultrasonic wave travelling on the sample surface. The height variation of the grating is represented by $g(x)$ and the surface acoustic wave is represented by $u(x-c t) . \quad k$ is the optical wavevector and the bar symbolizes the average.

The ultrasonic displacement can be represented as

$$
u(x, t)=A_{\mathrm{SAW}} \cos \left(k_{\mathrm{SAW}}(x-c t)\right),
$$

where $A_{\mathrm{SAW}}$ is the amplitude of the ultrasonic wave and $k_{\mathrm{SAW}}$ is its wavevector. The square grating can be described as a step function, hence

$$
\overline{\mathrm{e}^{2 \mathrm{i} k g(x)}}=\overline{\mathrm{e}^{2 \mathrm{i} k(0)}+\mathrm{e}^{2 \mathrm{i} k h}}=\frac{1+\mathrm{e}^{2 \mathrm{i} k h}}{2} .
$$

The actual signal on the photodetector would be

$$
i(t) \propto s(t) s^{*}(t),
$$

where $s^{*}(t)$ denotes the conjugate of $s(t)$. From equations (1), (3) and (4) it can be seen that for $h=\lambda / 8$ (where $\lambda$ is the wavelength of the detection laser) maximum interference signal is obtained.

Figure 7 shows simulations of the theoretical predictions for the optical detection of the signal due to the d-CHOT. We assumed $N_{d}$ to be 10 , a surface acoustic wave of $10 \mathrm{MHz}$ generated by a Gaussian laser pulse with an amplitude of $10 \mathrm{~nm}$, the detection laser beam spot size to be as big as the CHOT and the optical power of the detection laser to be $1 \mathrm{~mW}$. Figure $7(a)$ shows the reflected field of the optical probing beam and figure $7(b)$ shows the detected signal oscillations due to the SAW wave travelling on the sample surface.

For oblique incidence, the effective grating height is reduced by a factor of $\cos \theta$, where $\theta$ is the angle of incidence. In this case, the dc background due to the term describing the grating (3) is changed. Figure 8 shows three graphs for $0^{\circ}$, $35^{\circ}$ and $60^{\circ}$ angle of incidence. The peak-to-peak maximum power is $5.7 \mu \mathrm{W}, 5.5 \mu \mathrm{W}, 4.0 \mu \mathrm{W}$, respectively; therefore the incidence angle has a minimal effect on the detected signal.

\subsection{CHOT system}

Combining the mathematical analysis for the g-CHOT and the d-CHOT, it can be seen that when the two elements of the CHOT are brought together in one system for generation and detection, then the frequency bandwidth of the resulting signal will be the product of the two frequency contents.

\section{Experimental results}

Experiments have been performed to demonstrate the operation of the g-CHOT and the d-CHOT as narrowband transducers for generation and detection in a coupled system.

A BK7 glass sample of size $50 \mathrm{~mm} \times 50 \mathrm{~mm}$ and thickness $8 \mathrm{~mm}$ was used. The surface wave velocity of the sample was measured using the OSAM system [19] and was found to be $3370 \mathrm{~m} \mathrm{~s}^{-1}$. The CHOTs were manufactured by photolithography. The absorbing stripes of the g-CHOT were made by evaporating an aluminium coating of $\sim 318 \mathrm{~nm}$ thickness. The laser used as an actuator for the g-CHOT was a Qswitched Nd:YAG laser emitting at $1064 \mathrm{~nm}$ wavelength of pulse duration $20 \mathrm{~ns}, 300 \mathrm{~mW}$ of power and $1 \mathrm{kHz}$ repetition 


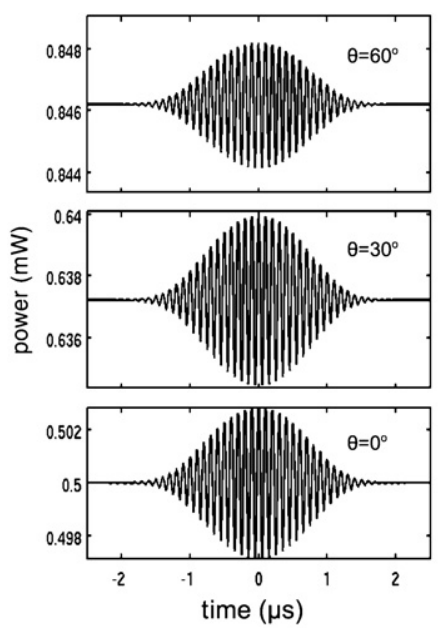

(a)

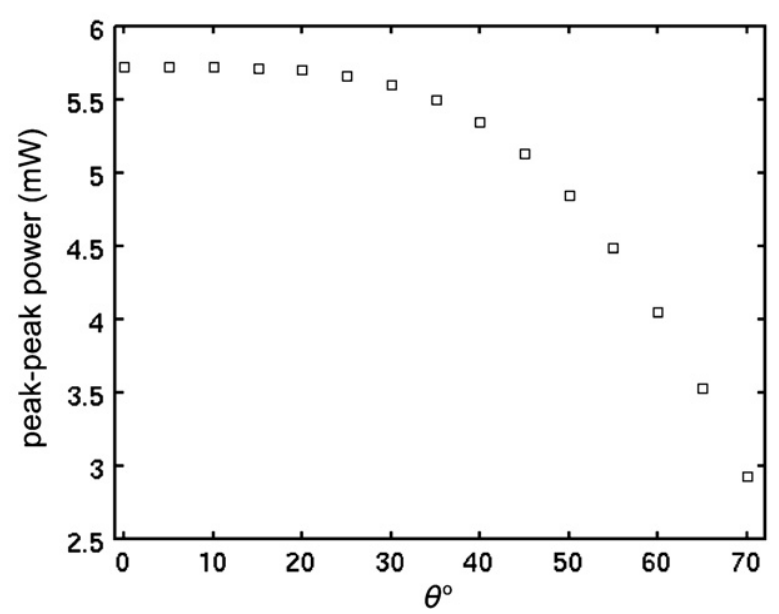

(b)

Figure 8. Simulations of optical power detection of ultrasonic signal from the d-CHOT for oblique angle of incidence. The numerical values used are the same as for figure 7 but the amplitude of the SAW was assumed to be 0.5 nm: $(a)$ detected power for $\theta=0^{\circ}, \theta=35^{\circ}$ and $\theta=60^{\circ}$. (b) Variation of peak-to-peak detected power versus angle of incidence.

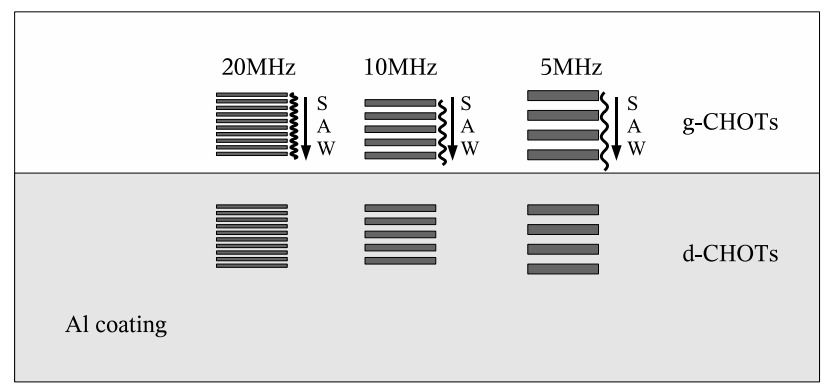

Figure 9. Schematic representation of coupled CHOTs.

rate corresponding to $0.3 \mathrm{~mJ}$ per pulse. The d-CHOT was made by evaporating a thin $(\sim 160 \mathrm{~nm})$ coating of aluminium initially, to make the surface reflective and then the stripes were made by a second layer of aluminium creating steps of $\sim 70 \mathrm{~nm}$ height. For optimum operation and at normal incidence on the d-CHOT, the height of its steps should have been $1 / 8$ of the detection laser wavelength. Since we were using a $\mathrm{HeNe}$ laser emitting at $633 \mathrm{~nm}$, the optimum step height should have been $79 \mathrm{~nm}$. Thus, we were not operating at optimum conditions. In addition, the angle of incidence was estimated to be $\sim 35^{\circ}$ due to the experimental setup, meaning that the path difference was $\sim 115 \mathrm{~nm}$ (path difference $=2($ step height $) \cos \theta)$ instead of $158 \mathrm{~nm}$. We can estimate the reduction of efficiency to be $\sin \left(\frac{115}{158} \frac{\pi}{2}\right) \sim 0.9$ of the optimum value. A single transverse mode $\left(\mathrm{TEM}_{00}\right) \mathrm{HeNe}$ laser was used, operating at $\mathrm{CW}$ mode and its power output was $10 \mathrm{~mW}$. It needs to be noted here that the temporal and spatial coherence of the detection light source are not particularly important and for the latter it should be of the order of $\lambda / 4$.

Three different pairs of CHOTs were made on the same sample. One with line (and spacing) thickness corresponding to the generation and detection of $5 \mathrm{MHz}$ SAWs (thickness = $336 \mu \mathrm{m}$ ), one for $10 \mathrm{MHz}$ SAWs (thickness $=168 \mu \mathrm{m}$ ) and one for $20 \mathrm{MHz}$ SAWs (thickness $=84 \mu \mathrm{m}$ ). The number of lines in each CHOT for the $5 \mathrm{MHz}, 10 \mathrm{MHz}$ and $20 \mathrm{MHz}$

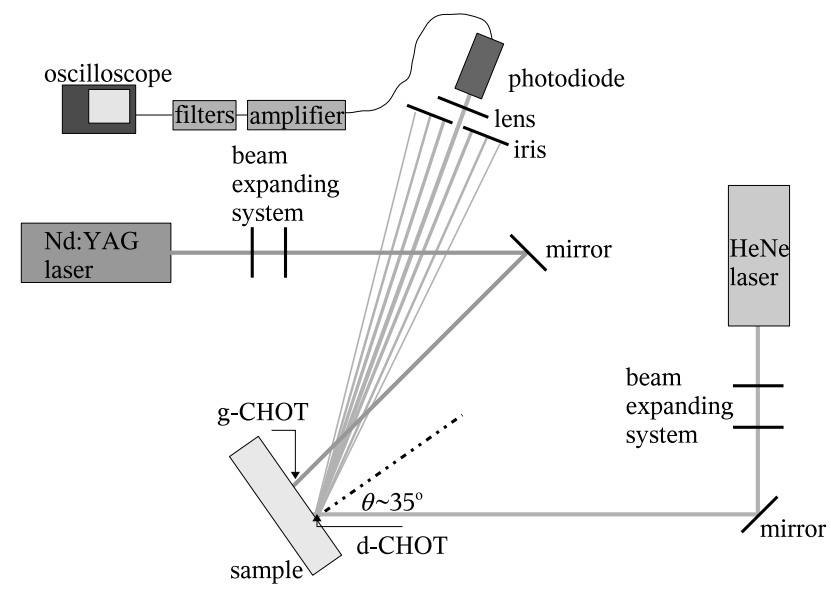

Figure 10. Experimental setup for the coupled CHOT system.

system, was 10, 20 and 40, respectively. Each CHOT was $0.8 \mathrm{~cm} \times 0.6 \mathrm{~cm}$ in size and the $\mathrm{g}$-CHOT was separated from the d-CHOT by $\sim 1.2 \mathrm{~cm}$. A representation of the sample surface can be seen in figure 9 .

The experimental setup is depicted in figure 10 . The spot size of the generating beam on the surface of the sample was controlled by a lens system and was $0.5 \mathrm{~cm}$ in diameter (i.e., $40 \%$ of the area of the g-CHOT) while the incident energy was kept constant at $300 \mathrm{~mW}$ at $1 \mathrm{kHz}$ repetition rate (corresponding to energy density of $1.53 \mathrm{~mJ} \mathrm{~cm}^{-2}$ ). The spot size of the detection beam was also $\sim 0.5 \mathrm{~cm}$ diameter. The various diffraction orders and the directly reflected beam (zero order) were separated by means of an iris and the remaining beam was then focused onto a photodiode. The signal was initially filtered with a $3 \mathrm{MHz}$ high pass filter and a $21.4 \mathrm{MHz}$ low pass filter. It was then amplified and finally captured on the oscilloscope. The captured waveforms can be seen in figure 11(a). The signal was averaged over 500 pulses. Figure 11(b) shows the frequency content of the corresponding signals. 

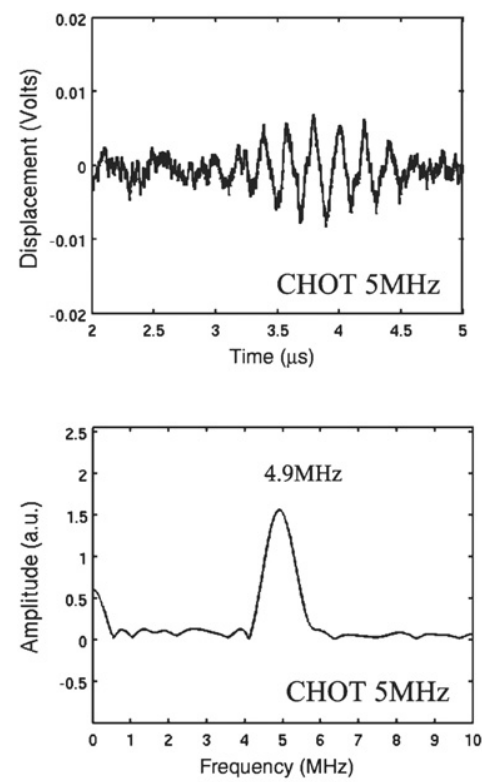

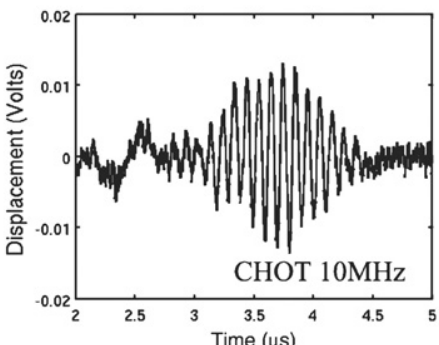

(a)

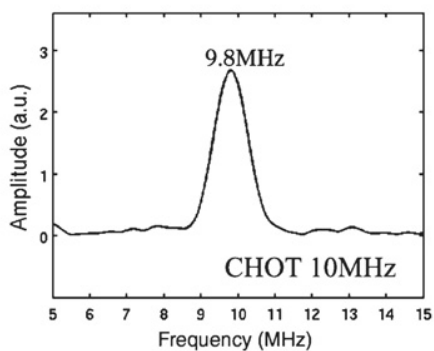

$(b)$
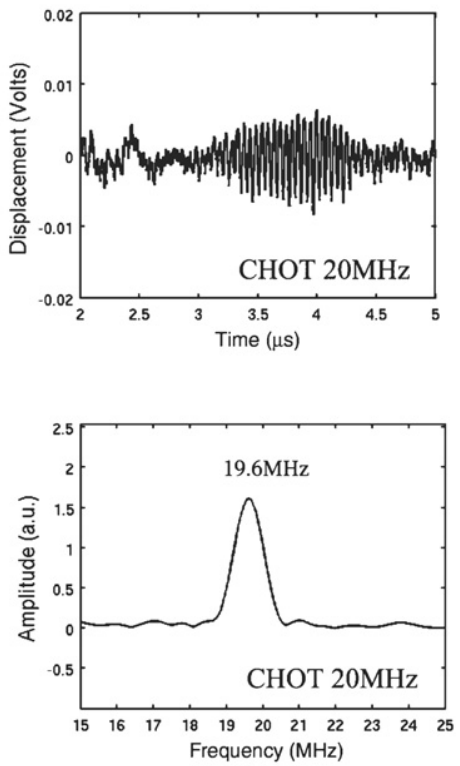

Figure 11. Experimental results: (a) waveforms captured using CHOT systems for 5, 10 and 20 MHz, averaged over 500 pulses, (b) frequency content of the respective waveforms.

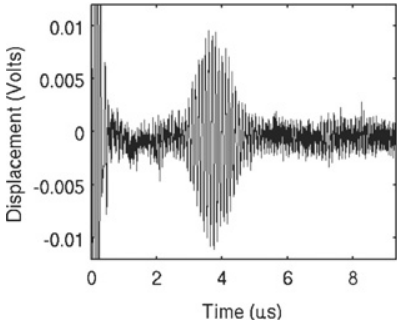

(a)

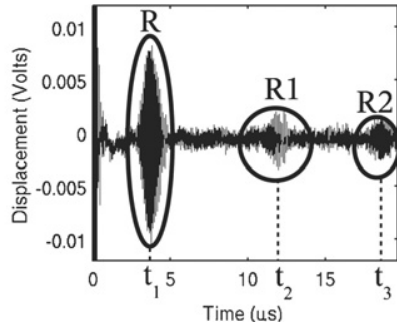

(b)

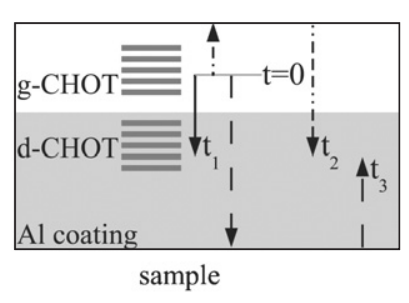

(c)

Figure 12. Experimental results: waveforms captured using a CHOT system for $10 \mathrm{MHz}$ and appropriate filtering. The electronic noise at the beginning of the graphs marks the beginning of the generation laser pulse. (a) Waveform averaged over 500 pulses, $(b)$ waveform averaged over 2000 pulses showing a larger time window. The signal marked as $\mathrm{R}$ is the first signal to be detected. The signals marked as $\mathrm{R} 1$ and $\mathrm{R} 2$ correspond to reflections from the top and the bottom edge of the sample, $(c)$ schematic representation of the sample showing the wave arrival times. The wave represented by the solid arrow corresponds to the first arrival (R), the one with the short dashes is the reflection from the top edge (R1) and the one with the long dashes is the reflection from the bottom edge (R2).

Figure 12 shows waveforms captured by the $10 \mathrm{MHz}$ CHOT system under similar experimental conditions as described above but with appropriate filtering (band pass 10.7 MHz). The electronic noise at the beginning of the graphs marks the beginning of the generation laser pulse. Figure 12(a) shows a waveform after 500 pulses averaging while figure 12(b) shows a waveform over a larger temporal window and averaged over 2000 pulses. The signals following the main trend of pulses are due to reflections of the ultrasound from the top and the bottom edges of the sample.

A special note should be made concerning the energy of the generating laser. For the experimental conditions described above and the laser pulse shown in figure 2, the incident power density $I_{0}$ was $\sim 7.7 \times 10^{8} \mathrm{~W} \mathrm{~m}^{-2}$. However, aluminium reflects $93 \%$ of this incident energy meaning that the absorbed power density is $\sim 5.4 \times 10^{7} \mathrm{~W} \mathrm{~m}^{-2}$. The aluminium stripes of the g-CHOT were relatively thin $(\sim 318 \mathrm{~nm})$ and concerns may arise about the thermal load on the substrate, especially if the substrate is a bad heat conductor. Using the equation from [20]:

$$
T(z, t)=\frac{2 I_{0}(\kappa t)^{1 / 2}}{K} \mathrm{ierfc} \frac{z}{2(\kappa t)^{1 / 2}}
$$

we have calculated the maximum temperature rise $(T)$ at a depth $(z)$ of $300 \mathrm{~nm}$ below the sample surface to be $0.3{ }^{\circ} \mathrm{C}$. The thermal conductivity $(K)$ and thermal diffusivity $(\kappa)$ values for aluminium were $240 \mathrm{~W} \mathrm{~m}^{-1} \mathrm{~K}^{-1}$ and $1.0 \times 10^{-4} \mathrm{~m}^{2} \mathrm{~s}^{-1}$ respectively, as taken from [21]. Equation (5) is valid for a laser pulse with top hat temporal and spatial beam profile, nevertheless it gives a good first approximation for the expected temperature rise.

Even if $100 \%$ of the laser energy were absorbed, the maximum temperature rise at a depth equal to the thickness of the aluminium layer would be $4{ }^{\circ} \mathrm{C}$, meaning that the thermal effects on both the aluminium and the substrate would be minimal even if the substrate were a bad heat conductor. 


\section{Conclusions and future work}

In this paper we have presented a new concept of optical transducers, the CHOTs, and shown experimental results from their operation. They consist of a generation (g-CHOT) and a detection (d-CHOT) part and can be used either as a coupled system or individually. Lasers are used as actuators providing remote and couplant-free operation. Their operation is based on minimal absorption (for the g-CHOT) or reflection (for the d-CHOT) modification of the surface of the inspected part.

For the g-CHOT, the pulsed laser is used as an actuator that provides a wide selection of frequencies limited only by its broadband frequency spectrum (dependent on the laser's pulse duration) and its energy. Thus, the user, by choosing the appropriate g-CHOT can have a wide selection of narrowband exciters. In our experiments we used a Nd:YAG laser emitting at $1064 \mathrm{~nm}$. Such lasers have been fully adapted in the industry because they have become portable, they need little maintenance and they are becoming continuously less expensive. The laser we used had a rise time of $10 \mathrm{~ns}$ (corresponding to a useful frequency content extending up to $\sim 30 \mathrm{MHz}$ ) and delivered enough energy to excite 5, 10 and $20 \mathrm{MHz}$ ultrasonic signals as demonstrated.

The d-CHOT is novel in the laser ultrasonic detection methods. It can be used on its own to detect ultrasound generated by other means but combined with the g-CHOT they make a powerful and versatile system. For the d-CHOT, a class 3R laser is suitable. We used a CW HeNe laser emitting at $633 \mathrm{~nm}$ and $10 \mathrm{~mW}$ of power. This proved sufficient for detecting the ultrasound generated by the g-CHOT. The d-CHOT takes advantage of the direct reflection of the laser beam and for this reason it does not have high demands on the reflectance of the sample surface. Even in 'noisy' environments, where the angle of incidence cannot be maintained with great accuracy, we have shown that this does not have a significant effect on the detected signal. Assuming that the diffracted orders are well separated and that the zero order can be isolated, the only tradeoff is the photodetector size which limits its time response.

In the immediate future we are planning to experiment with different manufacturing processes making the preparation of the CHOTs faster and cheaper but also to increase the efficiency of the CHOT system. One way of accomplishing this is by increasing the absorption contrast of the g-CHOT. Since the detected electrical signal power is proportional to the square of the absorbed laser power, if we achieved $100 \%$ absorption contrast (instead of $7 \%$ which is what we currently have), we would have 14 times more absorbed optical power corresponding to $\sim 200$ times better signal-to-noise ratio. We are also planning to experiment with different CHOT structures which would allow excitation of other wavemodes such as bulk waves but also control the directivity of the ultrasonic waves by generating/detecting for example focused waves.

One of the applications that CHOTs are most suitable for, is nonlinear elastic wave spectroscopy (NEWS). NEWS techniques have shown to be more sensitive in detecting microscale fractures and predicting early damage [22] than traditional linear acoustical methods. The concept of NEWSbased methods is that internal damage can be measured directly with the instantaneous detection of an increase in the nonlinearity parameters. In order for nonlinear measurements to be reliable, the ultrasonic generation and detection systems should be free from their own inherent nonlinearities and for the detection system in particular, it should have high sensitivity in order to receive the generally small signals associated with harmonics. The fact that CHOTs have a high $Q$ factor makes them ideal for such use where narrowband excitation and detection is of primary importance. Since one can control the frequency content of the generated/detected wave, harmonic cross talk can be prevented. For example, a g-CHOT can generate the fundamental chosen so that the harmonic lies outside the frequency content of the generating laser. The d-CHOT can then be used to detect and monitor the harmonic that is indicative of the presence or the development of a defect. In another example, a g-CHOT pattern combining two different frequencies (one high and one low) can be used in experiments of frequency mixing similar to those described in [23]. In this case the d-CHOT would detect the high frequency and the nonlinear parameter is measured by the phase modulation observed in the high frequency. We are currently in the process of realizing such experiments.

Among CHOTs' many advantages over traditional contact transducers is their low profile (minimal weight and size $<1 \mathrm{~cm}^{2}$ ) which allows minimal impact on the inspected sample. Thus, CHOTs can be permanently attached to the sample to provide reliable and repeatable measurements. Furthermore, they have the potential to become so cheap as to be thought of as disposable. Since the laser light can be delivered through fibres or extremely simple optical systems they are suitable for inspection of large structures while their remote use makes them ideal for hazardous environments. As the user has the freedom to choose the excited wavemode depending on the CHOT structure, they also have a great potential to find use as biological, gas and food sensors (where they could also be incorporated into packaging) not only as cheap alternatives to piezoelectric transducers but also because of their versatility, the fact that they can be used in large numbers and especially because of their low impact on the structures. Another very important application of CHOTs would be related to aerospace applications. In most cases, the area where a crack is most likely to appear is known. Scanning of the area is possible if the CHOT structures cover a large area of the inspected component. A CHOT system with a chosen frequency can be placed in the positions where failure is most likely and constantly monitor the component throughout its lifetime. Such an application would be particularly useful for places of limited access where a transducer cannot be placed or environments of extreme temperatures. The small size and cost of CHOTs systems will mean that multiple CHOTs can be placed on components allowing multipoint defect detection or scanning. For all these reasons it is expected that CHOTs will have a big impact in the field of nondestructive testing.

\section{Acknowledgments}

This work is supported by the EU through the AERONEWS research project (FP6-502927) and the EPSRC through the research project EP/C512375/1. 


\section{References}

[1] Bonello B and Ajinou A 2001 Surface acoustic waves in the $\mathrm{GHz}$ range generated by periodically patterned metallic stripes illuminated by an ultrashort laser pulse J. Acoust. Soc. Am. 110 1943-9

[2] Lin H-N, Maris H J, Freund L B, Lee K Y, Luhn H and Kern D P 1993 Study of vibrational modes of gold nanostructures by picosecond ultrasonics J. Appl. Phys. $7337-45$

[3] Hurley D H and Telschow K L 2002 Picosecond surface acoustic waves using a suboptical wavelength absorption grating Phys. Rev. B 66153301

[4] Lee T, Ohmori K, Shin C-S, Cahill D G, Petrov I and Greene J E 2005 Elastic constants of single-crystal $\operatorname{TiN}_{x}(001)(0.67 \leqslant x \leqslant 1.0)$ determined as a function of $x$ by picosecond ultrasonic measurements Phys. Rev. B 71144106

[5] Palik E D 1998 Handbook of Optical Constants of Solids (London: Academic)

[6] Huang J, Krishnaswamy S and Achenbach J D 1992 Laser generation of narrow-band surface waves J. Acoust. Soc. Am. 92 2527-31

[7] di Scalea F L, Berndt T P, Spicer J B and Djordjevic B B 1999 Remote laser generation of narrow-band surface waves through optical fibers IEEE Trans. Ultrason. Ferroelectr. Freq. Control 46 1551-7

[8] Jarzynski J and Berthelot Y H 1989 The use of optical fibers to enhance the laser generation of ultrasonic waves J. Acoust. Soc. Am. 85 158-62

[9] Doyle P A and Scala C M 1996 Near-field ultrasonic Rayleigh waves from a laser line source Ultrasonics 34 1-8

[10] Ash E A, Dieulesaint E and Rakouth H 1980 Generation of surface acoustic waves by means of a c.w. laser Electron. Lett. 16 470-2

[11] McKie A D W, Wagner J W, Spicer J B and Penney C M 1989 Laser generation of narrow-band and directed ultrasound Ultrasonics 27 323-30
[12] Wagner J W, Deaton J B Jr, McKie A D W and Spicer J B 1990 Laser ultrasonics: generation and detection considerations for improved signal-to-noise ratio Proc. SPIE 4915 491-501

[13] Auth D C 1970 New high-power source of coherent microwave phonons Appl. Phys. Lett. 16 521-3

[14] Cachier G 1970 Optical excitation of high-amplitude surface waves Appl. Phys. Lett. 17 419-21

[15] Edwards C, Bushell A C, Palmer S B and Nacano H 1992 Laser interference grating for surface wave generation Nondestr. Test. Eval. 10 15-23

[16] Liu Y-H, Wu T-T and Lee C-K 2002 Application of narrow band laser ultrasonics to the nondestructive evaluation of thin bonding layers J. Acoust. Soc. Am. $1112638-43$

[17] Clark M, Sharples S D and Somekh M G 2000 Fast all-optical Rayleigh wave microscope: imaging on isotropic and anisotropic material IEEE Trans. Ultrason. Ferroelectr. Freq. Control 47 65-74

[18] Hong Y, Sharples S D, Clark M and Somekh M G 2003 Rapid measurement of surface acoustic wave velocity on single crystals using an all-optical adaptive scanning acoustic microscope Appl. Phys. Lett. 83 3260-2

[19] Sharples S D, Clark M and Somekh M G 2005 Fast noncontact imaging of material microstructure using local surface acoustic wave velocity mapping Proc. 2005 IEEE Ultrason. Symp. pp 886-9

[20] Carslaw H S and Jaeger J C 1959 Conduction of Heat in Solids (Oxford: Oxford University Press)

[21] Scruby C B and Drain L E 1990 Laser Ultrasonics: Techniques and Applications (Bristol: Adam Hilger) p 227

[22] Bulk O, Morris W L and Richardson J M 1978 Acoustic harmonic generation at unbonded interfaces and fatigue cracks Appl. Phys. Lett. 33 371-3

[23] Vila M, Meulen F V, Dos Santos S, Haumesser L and Matar O B 2004 Contact phase modulation method for acoustic nonlinear parameter measurement in solid Ultrasonics 42 1062-5 